and triglyceride levels in white Europeans and to a lesser extent South Asians; opposite patterns were observed in black AfricanCaribbeans (likelihood-ratio tests for interactions between NS-SEC and ethnicity, all $\mathrm{p}<0.05)$. There were marked ethnic differences in diabetes risk markers. Compared to white Europeans, South Asian children had higher fat mass index (\% difference 7.3; 95\% CI 2.8 to 12.0), sum of skinfolds $(5.1 ; 1.1,9.4)$, HbA1c $(2.1 ; 1.6,2.7)$, glucose $(0.8 ; 0.2,1.5)$, insulin resistance $(29.6 ; 23.1,36.4)$, triglycerides (12.9; $9.4,16.5)$ and $C$ reactive protein $(43.3 ; 28.6,59.7)$ and lower HDLcholesterol $(-2.9 ;-1.3,-4.5)$. In contrast, black African/Caribbean children had less marked increases in $\mathrm{HbA1c}$, insulin resistance and $C$ reactive protein but conversely, had lower triglycerides and higher HDL-cholesterol; adiposity levels were not consistently increased. However, adjustment for socio-economic position had no material effect on the ethnic differences in metabolic markers observed.

Conclusions Although socio-economic position showed little overall association with diabetes risk markers in this multi-ethnic study population, there were appreciable associations within individual ethnic groups. Ethnic differences in socio-economic position did not explain marked ethnic differences in emerging risks of type 2 diabetes between South Asians, black African-Caribbeans and white Europeans; other explanations for these ethnic differences should be sought.

\section{INCIDENCE RATE TRENDS IN CHILDHOOD TYPE 1 DIABETES IN YORKSHIRE, 1978-2007: EFFECTS OF ETHNICITY AND AGE AT DIAGNOSIS}

doi:10.1136/jech.2010.120956.20

${ }^{1} \mathrm{~K}$ Harron, ${ }^{1} \mathrm{P}$ A McKinney, ${ }^{1} \mathrm{R}$ G Feltbower, ${ }^{1} \mathrm{C}$ R Stephenson, ${ }^{2} \mathrm{H} \mathrm{J}$ Bodansky, ${ }^{3} \mathrm{P}$ Norman, ${ }^{1} \mathrm{R}$ C Parslow. ${ }^{1}$ Paediatric Epidemiology Group, University of Leeds, UK; ${ }^{2}$ Leeds General Infirmary; ${ }^{3}$ School of Geography, University of Leeds, UK

Objective To examine incidence rates and trends of childhood Type 1 diabetes in Yorkshire from 1978 to 2007.

Methods Data from the population-based Yorkshire Register of Diabetes in Children and Young People was used to analyse the incidence of Type 1 diabetes in children aged $<15$ years diagnosed in the former Yorkshire Regional Health Authority. Incidence rates (per 100000 per year) were estimated using mid-year population estimates stratified by sex, age and ethnicity: south Asian (Indian, Pakistani, Bangladeshi) or non-south Asian (all other ethnicities). Ethnicity was assigned using two name recognition programs (Nam Pehchan and SANGRA) and a local expert. Age-sex standardised rates were calculated between 1978 and 2007 and by ethnic-group between 1990 and 2007. Poisson regression was used to assess incidence trends and estimate predicted rates up to 2020. Goodnessof-fit, AIC and likelihood-ratio tests were used to assess model fit.

Results 3896 children were diagnosed in Yorkshire between 1978 and 2007. Overall incidence was 18.1 (95\% CI 17.5 to to 18.6), increasing from 13.3 (1978 to 1987 ) to 16.9 (1988 to 1997$)$ to 24.1 (1998 to 2007). Incidence increased significantly over time: average annual percentage change (AAPC) was $2.8 \%$ (1.8 to 3.8 ). The inclusion of an age-sex interaction term provided evidence for differences in trends between sexes depending on age, with females having higher incidence and AAPC than males for those aged 5-9.

Overall incidence for non-south Asians (21.4; 20.6 to 22.3) was significantly higher than that of south Asians (14.6; 12.3 to 17.0) over the entire study period. A significant increasing trend in incidence was observed for non-south Asians of $3.3 \%$ (1.3 to 5.2) compared to a non-significant trend seen in south Asians $(1.9 \%$; -0.4 to 4.3). Overall forecasted incidence for 2020 is 38.3 per 100000 .

Conclusions Type 1 diabetes incidence rates have risen almost uniformly for non-south Asians of all ages but not for south Asians, contrary to findings in the Bradford area of Yorkshire between 1978 and 1998. Overall incidence increased most quickly in the 5-9 agegroup. Incidence doubled from 12.5 to 25.2 between 1978 and 2007. If current trends continue, rates will rise by $52 \%$ to 38.3 between 2007 and 2020.

\section{Tuesday 7 September 2010 Parallel Session B}

\section{Mental health \\ 021 RISK OF OVERDOSE MORTALITY DURING THE INITIAL 2 WEEKS AFTER ENTERING OR RE-ENTERING METHADONE TREATMENT IN SCOTLAND: RETROSPECTIVE COHORT STUDY}

doi:10.1136/jech.2010.120956.21

${ }^{1} \mathrm{G}$ Cousins, ${ }^{2} \mathrm{C}$ Teljeur, ${ }^{1} \mathrm{~N}$ Motterlini, ${ }^{3} \mathrm{C}$ McCowan, ${ }^{1} \mathrm{~T}$ Fahey, ${ }^{1} \mathrm{~B} \mathrm{D}$ Dimitrov. ${ }^{1} \mathrm{HRB}$ Centre for Primary Care Research, Royal College of Surgeons in Ireland, Dublin, Republic of Ireland; ${ }^{2}$ Department of Public Health and Primary Care, Trinity College Dublin, Republic of Ireland; ${ }^{3}$ Health Informatics Centre, University of Dundee, UK

Objectives Heroin users are at high risk of premature mortality. Despite the evidence supporting methadone maintenance programmes (MMT), methadone itself has been associated with drug-related deaths. This study aims to determine whether people prescribed methadone have an elevated risk of overdose mortality during periods of treatment transition, particularly during treatment initiation.

Method Retrospective cohort study of 3162 Scottish people prescribed and dispensed liquid methadone between January 1993 and February 2004. Observation time was defined as a period during methadone treatment or a period of maximum 6 months after leaving treatment. Individual observation time was censored after 6 months off-treatment. A person's observation time started again if they re-entered treatment after an off-treatment period. The main outcome measure was drug-related mortality by means of Cox-proportional hazards models during the 12 years of followup. Drug-related deaths occurring during treatment or within 3 days after last methadone prescription were considered as cases "on treatment". Fatalities occurring 4 days or more after leaving treatment were considered to be drug-related deaths "off treatment".

Results Overall 130 people died, with 51 deaths identified as drugrelated deaths (20 off treatment and 31 in treatment). Risk of drugrelated mortality was higher during treatment than off treatment (adjusted hazard ratio $11.17,95 \%$ CI 4.51 to 27.64). Inspection of timing of death showed that the risk of drug-related mortality was higher during the initial two weeks of treatment (adjusted hazard ratio $16.93,95 \%$ CI 5.17 to 55.46 ) compared to the risk of mortality off treatment. Similarly, retention in treatment for more than 3 weeks was associated with increased mortality relative to being off treatment (adjusted hazard ratio 9.97, 95\% CI 4.08 to 24.39). In relation to risk of mortality during treatment, being in treatment for $3-10$ weeks (adjusted hazard ratio $0.36,95 \%$ CI 0.15 to 0.85 ) or greater than 10 weeks (adjusted hazard ratio 0.13 , 95\% CI 0.04 to 0.39 ) was associated with a reduced risk of mortality compared to the initial two weeks on treatment. These effects were observed after adjusting for all or some of the following covariates; coprescribing of benzodiazepines, psychiatric admission, number of methadone treatments, overuse of methadone and urine testing, where appropriate.

Conclusion Excess mortality risk in the initial two weeks of methadone treatment indicates the need for more care in prescribing and monitoring of methadone when starting or restarting a patient on methadone maintenance therapy. 